Proceedings

\title{
Synthesis and Characterization of Various Amino Acid Derived Thiohydantoins ${ }^{\dagger}$
}

\author{
Petar Stanić ${ }^{1}$, Marija Živković ${ }^{2}$ and Biljana Šmit 1 ,* \\ 1 Department of Chemistry, Faculty of Science, University of Kragujevac, Radoja Domanovića 12, \\ 34000 Kragujevac, Serbia; petar.stanic@pmf.kg.ac.rs \\ 2 Faculty of Medical Sciences, University of Kragujevac, Svetozara Markovića 69, 34000 Kragujevac, Serbia; \\ mzivkovic@kg.ac.rs \\ * Correspondence: biljana.smit@pmf.kg.ac.rs \\ + Presented at the 22nd International Electronic Conference on Synthetic Organic Chemistry, 15 November- \\ 15 December 2018; Available Online: https://sciforum.net/conference/ecsoc-22.
}

Published: 14 November 2018

\begin{abstract}
Hydantoins and their sulfur containing analogues, thiohydantoins, are cyclic ureides that have attracted huge attention ever since their discovery. Most of them are biologically active compounds and several points of structural diversity have made them very synthetically attractive. Although substituents can be introduced to the hydantoin nucleus, most substituted hydantoins are synthesized from substrates already containing these groups, while forming the hydantoin nucleus. This is a common route to the synthesis of hydantoins and one of them is employed in this study. A series of 3-allyl-2-thiohydantoins is synthesized from various $\alpha$-amino acids in a reaction with allyl isothiocyanate. The substitution of the acquired thiohydantoin depends on the structure of the starting $\alpha$-amino acid. The residual group of the $\alpha$-amino acid becomes the substituent at the C5position, while N-monosubstituted amino acids give rise to a substituent in the N1-position. The reaction is carried out in a two-step process and the reaction conditions generally depend on the nature of the amino acid itself. All thiohydantoins are obtained in a good yield and fully characterized by NMR and IR spectroscopy, as well as X-ray crystallography.
\end{abstract}

Keywords: thiohydantoins; synthesis; amino acids; substitution

\section{Introduction}

Hydantoins represent a large group of synthetically and biologically attractive compounds [1]. Structurally, they are five-membered cyclic ureides with several points of structural diversity (Figure 1) that give them interesting physical, chemical and biological properties [2].
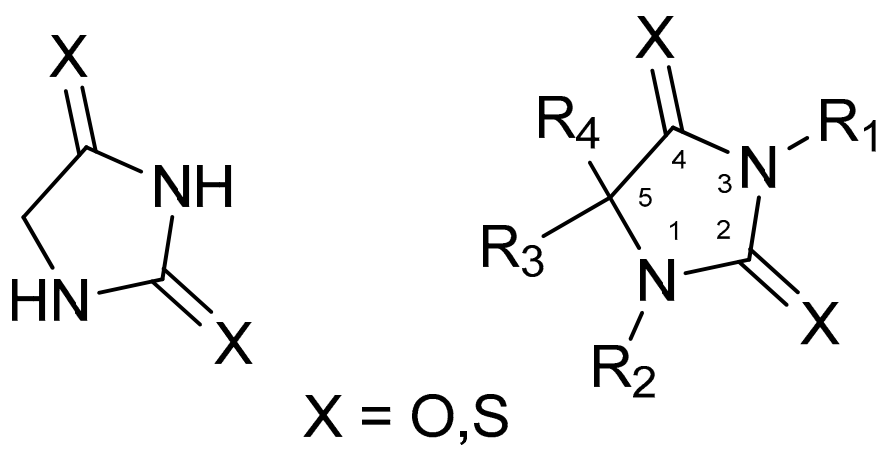

Figure 1. The structure of hydantoins and their derivatives. 
There are many synthetic routes to hydantoins. Although hydantoin derivatives can be synthesized by introducing substituents to the hydantoin nucleus, the most common route is synthesis from substrates that already contain the desired groups, while forming the hydantoin nucleus. One such route is the Bucherer-Bergs reaction, which involves aldehydes and ketones [3]. Another, perhaps more important in a physiological point of view, is the synthesis of hydantoins from amino acids, which are ever-present in the food chain and urea or thiourea [4]. This reaction is responsible for the occurrence of hydantoins in urine when protein consumption is increased. One more important route to hydantoin derivatives is the synthesis from $\alpha$-amino acids and alkyl or aryl isocyanates and isothiocyanates [5]. This route is employed in this study.

Aside from them being synthetically attractive, hydantoins exhibit a wide range of biological activity [6-9]. Some of the attributed biological properties include antimicrobial, antitumor, antiandrogen, antiteratogenic, hypnotic, antiepileptic and anticonvulsant activity, wound healing, muscle relaxant, treatment of cachexia, psoriasis, chorea, anoxia, tuberculosis and some infectious diseases.

Considering the plethora of their biological activities in this paper, we present the synthesis of a series of amino acid derived 3-allyl-2-thiohydantoins.

\section{Results and Discussion}

Eleven 2-thiohydantoin derivatives were synthesized from various $\alpha$-amino acids and allyl isothiocyanate (Scheme 1) in moderate to high yields (Table 1) according to a slightly modified previously reported procedure [10]. The synthesis is carried out in a two-step process and the reaction conditions generally depend on the nature of the amino acid itself. Amino acids $1 \mathrm{a}, 1 \mathrm{e}, 1 \mathrm{f}$ and $1 \mathrm{j}$ needed higher temperature and chloroform is used instead of methylene chloride. All obtained thiohydantoins are fully characterized by NMR and IR spectroscopy, as well as X-ray crystallography (Figure 2). Thiohydantoins $\mathbf{3 a}-\mathbf{3 f}$ are already known compounds, while $\mathbf{3 g}-\mathbf{3 k}$ are novel.<smiles>[R]C(N)C(=O)O</smiles>

1a-k<smiles>CO[C@H](C)Cl</smiles><smiles>[R]C(N)C(=O)OC</smiles>

$2 a-k$<smiles>C=CCN(CC)OCC</smiles><smiles>[Li]</smiles><smiles>[R]C1CCCN(CC=C)C(=S)N1</smiles>

3a-k

Scheme 1. The synthesis of amino acid derived 3-allyl-2-thiohydantoins.

The reaction presented in this work represents a convenient way to synthesize various substituted 3-allyl-2-thiohydantoins, the substitution of which generally depends on the nature of the starting $\alpha$-amino acid. The residual group of the $\alpha$-amino acid becomes the substituent at the C5position, while N-monosubstituted amino acids give rise to a substituent in the N1-position. As there are many substrates to choose from, including natural and unnatural $\alpha$-amino acids and also various isothiocyanates, many differently substituted thiohydantoins can be obtained with different chemical and biological properties.

This is important not only for fundamental research and a better understanding of hydantoin chemistry, but also for the search for compounds with potential medicinal applications. These compounds will be subjected to extensive biological evaluation. Additionally, they are suitable for further derivatization leading to more complex compounds with possibly new chemical properties and biological activities. 


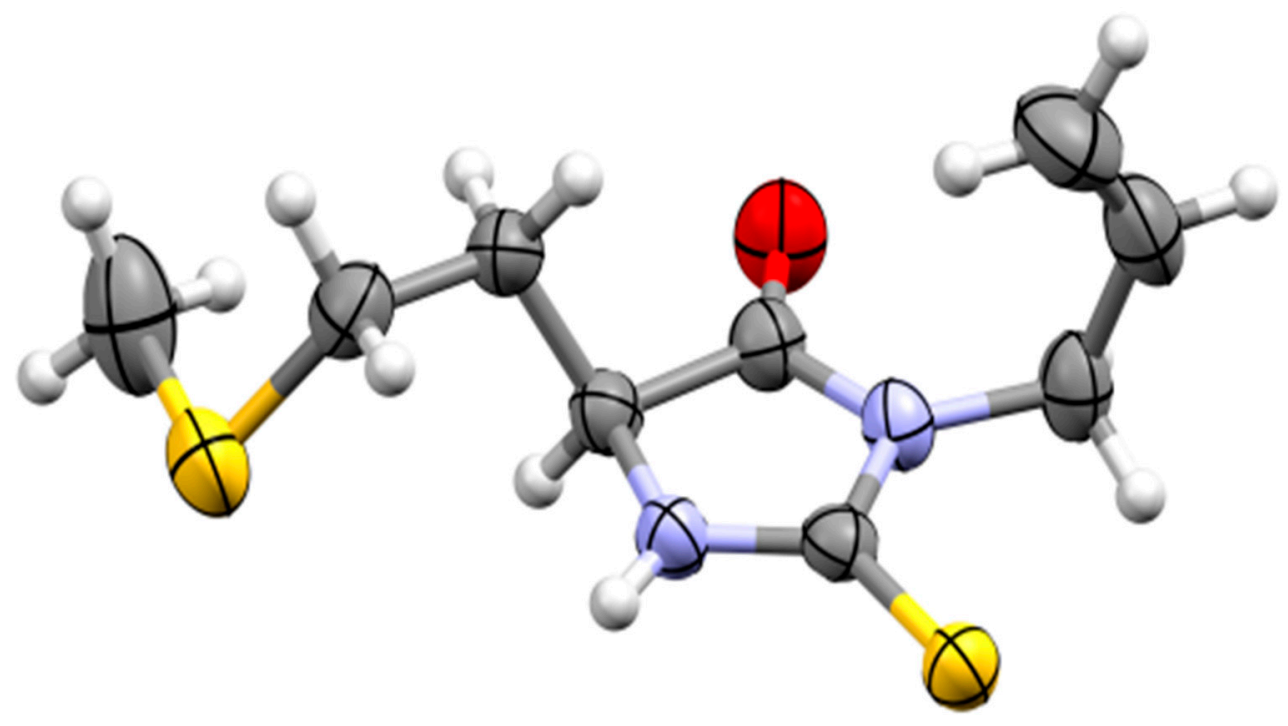

Figure 2. The ORTEP representation of thiohydantoin $3 \mathbf{h}$.

Table 1. The synthesis of amino acid derived 3-allyl-2-thiohydantoins.

\begin{tabular}{|c|c|c|c|}
\hline Entry & Substrate & Product & Yield (\%) \\
\hline a & & & 60 \\
\hline b & & & 51 \\
\hline c & & & 81 \\
\hline d & & & 84 \\
\hline e & & & 81 \\
\hline f & & & 51 \\
\hline g & & & 86 \\
\hline h & & & 82 \\
\hline $\mathbf{i}$ & & & 92 \\
\hline
\end{tabular}




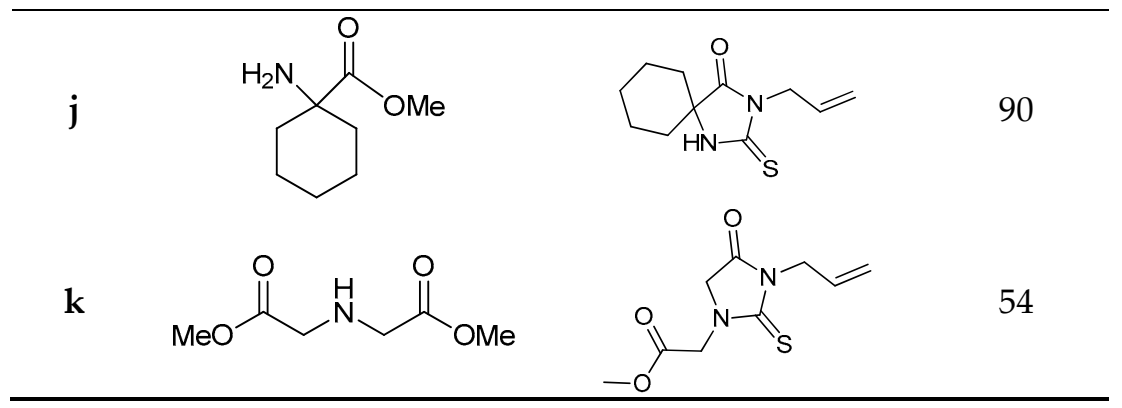

\section{Experimental}

\subsection{General}

All chemicals and reagents are commercially available and were used as received without further purification. Solvents were purified by distillation prior use. Anhydrous methanol was prepared by standard drying procedure.

Thin-layer chromatography (TLC) was performed on silica gel on A1 plates, layer thickness 0.2 $\mathrm{mm}$. IR spectra were recorded on a Perkin-Elmer FT-IR spectrometer model Spectrum One. ${ }^{1} \mathrm{H}$ and ${ }^{13} \mathrm{C}$ NMR spectra were recorded on a Varian Gemini $2000 \mathrm{NMR}$ spectrometer in $\mathrm{D}_{2} \mathrm{O}$ or $\mathrm{CDCl}_{3}$ as solvents. X-ray crystallographic analysis were performed on an Oxford Diffraction Gemini S diffractometer.

\subsection{General Procedure for the Preparation of the Amino Acid Methyl Esters 2a-k}

Amino acid methyl esters were prepared according to a well-known methanolic $\mathrm{HCl}$ method. 5 $\mathrm{mL}$ of methanol was added to a round bottom flask and cooled to $0^{\circ} \mathrm{C}$. Acetyl chloride $(2 \mathrm{~mL})$ was added slowly to the stirred solution and then stirred for another $20 \mathrm{~min}$ at $0^{\circ} \mathrm{C}$ to generate methanolic $\mathrm{HCl}$. An amino acid $(5 \mathrm{mmol})$ was added in one portion and the reaction was stirred overnight at room temperature. The solvent was removed in vacuo and solid amino acid methyl ester hydrochloride (yields ranging from 88 to $96 \%$ ) was used without further purification. Successful esterification was confirmed by ${ }^{1} \mathrm{H}$ NMR spectroscopy.

\subsection{General Procedure for the Preparation of the Amino Acid Derived 2-Thiohydantoins $\mathbf{3} \mathbf{a}-\mathbf{k}$}

A mixture of $5 \mathrm{mmol}$ amino acid methyl ester hydrochloride, $5 \mathrm{mmol} \mathrm{Et} 3 \mathrm{~N}$ and $15 \mathrm{~mL}$ of $\mathrm{CH}_{2} \mathrm{Cl}_{2}$ or $\mathrm{CHCl}_{3}$ was stirred for about $20 \mathrm{~min}$ at room temperature until all of the ester was dissolved. Allyl isothiocyanate $(5 \mathrm{mmol})$ was added dropwise and the reaction mixture was heated under reflux for $7 \mathrm{~h}$. The solution was cooled at room temperature and the solvent was removed in vacuo. The residue was dissolved in $\mathrm{CH}_{2} \mathrm{Cl}_{2}$, washed with water and brine and dried over anhydrous $\mathrm{Na}_{2} \mathrm{SO}_{4}$. The solvent was once again removed in vacuo, leaving a crude solid product that was recrystallized from $\mathrm{CH}_{2} \mathrm{Cl}_{2} /$ hexane.

3-allyl-2-thioxoimidazolidin-4-one (3a). Brownish-yellow rod-like crystals; IR (KBr) v $\max$ : 3225, 2923, 1751, 1650, 1526, 1430, 1343, 1259, 1173, 929, $697 \mathrm{~cm}^{-1}$; ${ }^{1} \mathrm{H}$ NMR $\left(200 \mathrm{MHz}, \mathrm{CDCl}_{3}\right) \delta 4.17(\mathrm{~d}, J=1.4 \mathrm{~Hz}$, $2 \mathrm{H}), 4.37(\mathrm{dt}, J=1.2$ and $6.0 \mathrm{~Hz}, 2 \mathrm{H}), 4.99-5.35(\mathrm{~m}, 2 \mathrm{H}), 5.74-5.97(\mathrm{~m}, 1 \mathrm{H}), 7.78(\mathrm{bs}, 1 \mathrm{H}) \mathrm{ppm} ;{ }^{13} \mathrm{C} \mathrm{NMR}$ $\left(50 \mathrm{MHz}, \mathrm{CDCl}_{3}\right) \delta 43.3,48.5,118.7,130.5,171.7,184.7 \mathrm{ppm}$.

3-allyl-5-methyl-2-thioxoimidazolidin-4-one (3b). Yellowish needle crystals; IR (KBr) $v_{\max }$ :3170, 3012, 2920, 1743, 1647, 1538, 1429, 1346, 1263, 1171, 927, $636 \mathrm{~cm}^{-1}$; $1 \mathrm{H}$ NMR $\left(200 \mathrm{MHz}, \mathrm{CDCl}_{3}\right) \delta 1.49$ (d, J= $6.8 \mathrm{~Hz}, 3 \mathrm{H}), 4.21(\mathrm{q}, J=6.8 \mathrm{~Hz}, 1 \mathrm{H}), 4.43(\mathrm{dt}, J=1.2$ and $6.0 \mathrm{~Hz}, 2 \mathrm{H}), 5.19-5.32(\mathrm{~m}, 2 \mathrm{H}), 5.76-5.98(\mathrm{~m}$, $1 \mathrm{H}), 7.22$ (bs, 1H) ppm; ${ }^{13} \mathrm{C}$ NMR (50 MHz, $\left.\mathrm{CDCl}_{3}\right) \delta$ 11.0, 43.3, 55.0, 118.5, 130.6, 174.1, 183.5 ppm.

3-allyl-5-isopropyl-2-thioxoimidazolidin-4-one (3c). Yellowish needle crystals; IR (KBr) vmax: 3292, 3093, $2963,1725,1648,1512,1428,1355,1254,1171,929,664 \mathrm{~cm}^{-1} ; 1 \mathrm{H}$ NMR $\left(200 \mathrm{MHz}, \mathrm{CDCl}_{3}\right) \delta 0.94(\mathrm{~d}, J=$ $6.8 \mathrm{~Hz}, 3 \mathrm{H}), 1.08(\mathrm{~d}, J=6.8 \mathrm{~Hz}, 3 \mathrm{H}), 2.19-2.38(\mathrm{~m}, 1 \mathrm{H}), 4.00(\mathrm{dd}, J=1.4$ and $2.0 \mathrm{~Hz}, 1 \mathrm{H}), 4.42(\mathrm{~d}, J=5.6$ 
$\mathrm{Hz}, 2 \mathrm{H}), 5.18-5.32(\mathrm{~m}, 2 \mathrm{H}), 5.73-5.96(\mathrm{~m}, 1 \mathrm{H}), 7.61(\mathrm{bs}, 1 \mathrm{H}) \mathrm{ppm} ;{ }^{13} \mathrm{C} \mathrm{NMR}\left(50 \mathrm{MHz}, \mathrm{CDCl}_{3}\right) \delta 16.3$, $18.8,30.9,43.1,64.6,118.5,130.6,173.1,184.0 \mathrm{ppm}$.

3-allyl-5-isobutyl-2-thioxoimidazolidin-4-one (3d). White tiny needle crystals; IR (KBr) vmax: 3181, 3006, 2956, 1754, 1650, 1534, 1433, 1346, 1253, 1175, 926, $656 \mathrm{~cm}^{-1} ; 1 \mathrm{H}$ NMR $\left(200 \mathrm{MHz}, \mathrm{CDCl}_{3}\right) \delta 0.98(\mathrm{~d}, J=$ $6.0 \mathrm{~Hz}, 3 \mathrm{H}), 1.52-1.90(\mathrm{~m}, 3 \mathrm{H}), 4.14(\mathrm{dd}, J=2.6$ and $9.6 \mathrm{~Hz}, 1 \mathrm{H}), 4.42(\mathrm{dd}, J=1.2$ and $5.6 \mathrm{~Hz}, 2 \mathrm{H}), 5.19$ $5.30(\mathrm{~m}, 2 \mathrm{H}), 5.75-5.97(\mathrm{~m}, 1 \mathrm{H}), 7.78(\mathrm{bs}, 1 \mathrm{H}) \mathrm{ppm} ;{ }^{13} \mathrm{C} \mathrm{NMR}\left(50 \mathrm{MHz}, \mathrm{CDCl}_{3}\right) \delta$ 21.5, 23.0, 25.2, 40.4, 43.2, 58.0, 118.4, 130.5, 174.0, $183.5 \mathrm{ppm}$.

3-allyl-5-benzyl-2-thioxoimidazolidin-4-one (3e). White tiny needle crystals; IR (KBr) v $v_{\max }$ : 3205, 3033, 2920, 1749, 1647, 1524, 1428, 1344, 1250, 1175, 931, 732, $651 \mathrm{~cm}^{-1} ;{ }^{1} \mathrm{H}$ NMR $\left(200 \mathrm{MHz}, \mathrm{CDCl}_{3}\right) \delta 2.89$ $(\mathrm{dd}, J=9.0$ and $14.0 \mathrm{~Hz}, 1 \mathrm{H}), 3.33(\mathrm{dd}, J=3.6$ and $14.0 \mathrm{~Hz}, 1 \mathrm{H}), 4.31(\mathrm{~d}, J=3.8 \mathrm{~Hz}, 1 \mathrm{H}), 4.36(\mathrm{dd}, J=1.6$ and $5.6 \mathrm{~Hz}, 2 \mathrm{H}), 5.01-5.19(\mathrm{~m}, 2 \mathrm{H}), 5.61-5.82(\mathrm{~m}, 1 \mathrm{H}), 7.18-7.40(\mathrm{~m}, 6 \mathrm{H}) \mathrm{ppm} ;{ }^{13} \mathrm{C}$ NMR $(50 \mathrm{MHz}$, $\left.\mathrm{CDCl}_{3}\right) \delta 37.6,43.2,60.4,118.4,127.7,129.1,130.4,134.6,172.7,183.5 \mathrm{ppm}$.

3-allyl-5-(4-hydroxybenzyl)-2-thioxoimidazolidin-4-one (3f). Yellow tiny crystals; IR (KBr) v $\max$ : 3258, 3013, 2925, 1726, 1650, 1528, 1437, 1263, 1171, 960, $653 \mathrm{~cm}^{-1} ;{ }^{1} \mathrm{H}$ NMR $\left(200 \mathrm{MHz}, \mathrm{CDCl}_{3}\right) \delta 2.84(\mathrm{dd}, J=8.6$ and $14.0 \mathrm{~Hz}, 1 \mathrm{H}), 3.23(\mathrm{dd}, J=3.6$ and $14.0 \mathrm{~Hz}, 1 \mathrm{H}), 4.28(\mathrm{ddd}, J=0.8,3.8$ and $8.6 \mathrm{~Hz}, 1 \mathrm{H}), 4.34(\mathrm{dt}, J=$ 4.0 and $5.4 \mathrm{~Hz}, 2 \mathrm{H}), 5.0(\mathrm{bs}, 1 \mathrm{H}), 5.00-5.19(\mathrm{~m}, 2 \mathrm{H}), 5.63-5.82(\mathrm{~m}, 1 \mathrm{H}), 6.78(\mathrm{~d}, J=6.4 \mathrm{~Hz}, 2 \mathrm{H}), 7.07(\mathrm{~d}$, $J=6.4 \mathrm{~Hz}, 3 \mathrm{H}$ ) ppm; ${ }^{13} \mathrm{C}$ NMR $\left(50 \mathrm{MHz}, \mathrm{CDCl}_{3}\right) \delta 38.7,43.2,60.6,115.9,118.3,126.5,130.4,155.2,172.7$, $183.5 \mathrm{ppm}$.

3-allyl-5-((methylthio)methyl)-2-thioxoimidazolidin-4-one (3g). Light orange needle crystals; IR (KBr) v $v_{\text {max: }}$ 3182, 3087, 2915, 1743, 1648, 1526, 1427, 1343, 1254, 1175, 921, $639 \mathrm{~cm}^{-1} ;{ }^{1} \mathrm{H}$ NMR $\left(200 \mathrm{MHz}, \mathrm{CDCl}_{3}\right) \delta$ $2.19(\mathrm{~s}, 3 \mathrm{H}), 2.73(\mathrm{dd}, J=9.4$ and $14.0 \mathrm{~Hz}, 1 \mathrm{H}), 3.11(\mathrm{dd}, J=3.6$ and $14.0 \mathrm{~Hz}, 1 \mathrm{H}), 4.29(\mathrm{dd}, J=3.4$ and $8.2 \mathrm{~Hz}, 1 \mathrm{H}), 4.23(\mathrm{~d}, J=5.4 \mathrm{~Hz}, 2 \mathrm{H}), 5.19-5.34(\mathrm{~m}, 2 \mathrm{H}), 5.75-5.98(\mathrm{~m}, 1 \mathrm{H}), 7.41$ (bs, $1 \mathrm{H}) \mathrm{ppm} ;{ }^{13} \mathrm{C}$ NMR $\left(50 \mathrm{MHz}, \mathrm{CDCl}_{3}\right) \delta 16.2,35.8,43.4,58.5,118.6,130.4,172.2,183.7 \mathrm{ppm}$.

3-allyl-5-((methylthio)ethyl)-2-thioxoimidazolidin-4-one (3h). Light orange needle crystals; IR (KBr) v $v_{\max }$ : $3169,3002,2921,1741,1646,1531,1432,1346,1255,1165,923,650 \mathrm{~cm}^{-1} ;{ }^{1} \mathrm{H}$ NMR $\left(200 \mathrm{MHz}, \mathrm{CDCl}_{3}\right) \delta$ 2.01 (septet, $J=6.8 \mathrm{~Hz}, 1 \mathrm{H}), 2.12(\mathrm{~s}, 3 \mathrm{H}), 2.18-2.34(\mathrm{~m}, 1 \mathrm{H}), 2.68(\mathrm{t}, J=7.4 \mathrm{~Hz}, 2 \mathrm{H}), 4.28(\mathrm{ddd}, J=1.2$, 4.2 and $7.4 \mathrm{~Hz}, 1 \mathrm{H}), 4.43(\mathrm{~d}, J=6.0 \mathrm{~Hz}, 2 \mathrm{H}), 5.18-5.31(\mathrm{~m}, 2 \mathrm{H}), 5.76-5.97(\mathrm{~m}, 1 \mathrm{H}), 7.81(\mathrm{bs}, 1 \mathrm{H}) \mathrm{ppm}$; ${ }^{13} \mathrm{C}$ NMR (50 MHz, $\mathrm{CDCl}_{3}$ ) $\delta 15.3,30.3,30.4,43.3,58.5,118.6,130.5,173.4,183.6$ ppm.

3-allyl-5-((ethylthio)ethyl)-2-thioxoimidazolidin-4-one (3i). Yellowish needle crystals; IR (KBr) $v_{\max }$ : 3310, 3085, 2924, 1724, 1648, 1510, 1432, 1354, 1254, 1191, 930, $625 \mathrm{~cm}^{-1} ;{ }^{1} \mathrm{H}$ NMR $\left(200 \mathrm{MHz}, \mathrm{CDCl}_{3}\right) \delta 1.27$ $(\mathrm{t}, J=7.2 \mathrm{~Hz}, 3 \mathrm{H}), 2.00$ (septet, $J=6.4 \mathrm{~Hz}, 1 \mathrm{H}), 2.19-2.36(\mathrm{~m}, 1 \mathrm{H}), 2.60(\mathrm{q}, J=7.2 \mathrm{~Hz}, 2 \mathrm{H}), 2.71(\mathrm{t}, J=6.8$ $\mathrm{Hz}, 2 \mathrm{H}), 4.32(\mathrm{dd}, J=4.8$ and $8.2 \mathrm{~Hz}, 1 \mathrm{H}), 4.42(\mathrm{~d}, J=5.6 \mathrm{~Hz}, 2 \mathrm{H}), 5.19-5.30(\mathrm{~m}, 2 \mathrm{H}), 5.76-5.94(\mathrm{~m}, 1 \mathrm{H})$, 8.22 (bs, 1H) ppm; ${ }^{13} \mathrm{C} \mathrm{NMR}\left(50 \mathrm{MHz}, \mathrm{CDCl}_{3}\right) \delta 14.5,25.8,27.9,38.8,43.2,58.5,118.5,130.5,173.5,183.4$ ppm.

3-allyl-2-thioxo-1,3-diazaspiro[4,5]decan-4-one (3j). Brownish orange four-sided platy crystals; IR (KBr) $v_{\max }: 3271,3180,2939,1745,1716,1651,1508,1427,1215,1099,930,642 \mathrm{~cm}^{-1} ;{ }^{1} \mathrm{H} \mathrm{NMR}\left(200 \mathrm{MHz}, \mathrm{CDCl}_{3}\right)$ $\delta 1.12-1.98(\mathrm{~m}, 10 \mathrm{H}), 4.42(\mathrm{dt}, J=1.6$ and $4.0 \mathrm{~Hz}, 2 \mathrm{H}), 5.15-5.26(\mathrm{~m}, 2 \mathrm{H}), 5.78-5.95(\mathrm{~m}, 1 \mathrm{H}), 8.71(\mathrm{bs}$, $1 \mathrm{H}) \mathrm{ppm} ;{ }^{13} \mathrm{C}$ NMR $\left(50 \mathrm{MHz}, \mathrm{CDCl}_{3}\right) \delta 21.6,24.4,33.0,43.0,64.3,117.9,130.7,176.4,182.0 \mathrm{ppm}$.

Methyl 2-(3-allyl-4-oxo-2-thioxoimidazolidin-1-yl) acetate (3k). Yellow tiny crystals; IR (KBr) vmax: 3271, 3079, 2955, 1751, 1646, 1493, 1352, 1233, 1164, 940, $645 \mathrm{~cm}^{-1}$; ${ }^{1} \mathrm{H} \mathrm{NMR}\left(200 \mathrm{MHz}, \mathrm{CDCl}_{3}\right) \delta 3.80(\mathrm{~s}, 3 \mathrm{H})$, $4.18(\mathrm{~s}, 2 \mathrm{H}), 4.46(\mathrm{~d}, J=7.6 \mathrm{~Hz}, 2 \mathrm{H}), 4.62(\mathrm{~s}, 2 \mathrm{H}), 5.17-5.33(\mathrm{~m}, 2 \mathrm{H}), 5.75-5.98(\mathrm{~m}, 1 \mathrm{H}) \mathrm{ppm} ;{ }^{13} \mathrm{C}$ NMR (50 MHz, $\left.\mathrm{CDCl}_{3}\right) \delta, 44.4,47.4,52.4,52.6,118.5,130.5,167.9,169.8,184.1 \mathrm{ppm}$.

\section{Conclusions}

A series of eleven amino acid derived 3-allyl-2-thiohydantoins has been synthesized in good yields, five of which are novel. A convenient method for synthesis of various 2-thiohydantoin derivatives is described. An extensive biological evaluation will be done on the synthesized compounds. Additionally, since these compounds have functional groups in the side chains, further derivatization will be performed. As hydantoins represent a large group of biologically active and 
attractive compounds, some of which are already in use as drugs, this work will serve as a useful footnote in the search for more biologically active and potentially applicable compounds.

Acknowledgments: The authors are grateful to the Ministry of Education, Science and Technological Development of the Republic of Serbia (Project numbers 172016, 172034 and 172036) for financial support.

\section{References}

1. Ware, E. The chemistry of the hydantoins. Chem. Rev. 1950, 46, 403-470.

2. Konnert, L.; Lamaty, F.; Martinez, J.; Colacino, E. Recent advances in the synthesis of hydantoins: The state of the art of a valuable scaffold. Chem. Rev. 2017, 117, 13757-13809.

3. Bucherer, H.T.; Lieb, V.A. Über die bildung substituierter hydantoine aus aldehyden und ketonen. Synthese von hydantoinen. J. Prakt. Chem. 1934, 141, 5-43.

4. Heintz, W.Ueber ein Aethylderivat des Hydatoïns und die Bildung der Hydantoïnsäure aus Glycocoll. Justus Liebigs Annalen der Chemie 1865, 133, 65-74.

5. Marckwald, W.; Neumark, M.; Stelzner, R. Ueber Thiohydantoine und von diesen derivirende Basen. Gesellschaft 1891, 24, 3278-3298.

6. Ogawa, J.; Shimizu, S. Diversity and versatility of microbial hydantoin-transforming enzymes. J. Mol. Catal. B Enzym. 1997, 2, 163-176.

7. Trišović, N.P.; Ušćumlić, G.S.; Petrović, S.D. Hydantoins: Synthesis, properties and anticonvulsant activity. Hemijska Industrija 2009, 63, 17-31.

8. Rajic, Z.; Zorc, B.; Raic-Malic, S.; Ester, K.; Kralj, M.; Pavelic, K.; Balzarini, J.; de Clercq, E.; Mintas, M. Hydantoin Derivatives of L- and D-amino acids: Synthesis and Evaluation of Their Antiviral and Antitumoral Activity. Molecules 2006, 11, 837-848.

9. Ahmad, R.; Jabeen, R.; Zia-ul-Haq, M.; Nadeem, H.; Duddeck, H.; Verspohl, E.J. Chiral aryl sulfonyl Hydantoins as hypoglycemic agents. Z. Naturforsch. 2000, 55b, 203-207.

10. Reyes, S.; Burgess, K. On formation of thiohydantoins from amino acids under acylation conditions. J. Org. Chem. 2006, 71, 2507-2509.

(C) 2019 by the authors. Licensee MDPI, Basel, Switzerland. This article is an open access article distributed under the terms and conditions of the Creative Commons Attribution (CC BY) license (http://creativecommons.org/licenses/by/4.0/). 